\title{
The Spotted Li abundance on the surface of the CP star HD 3980
}

\author{
N.A. Drake ${ }^{1,2}$, N.S. Polosukhina ${ }^{3}$, R. de la Reza ${ }^{1}$ and M. Hack ${ }^{4}$ \\ ${ }^{1}$ Observatório Nacional/MCT, Rua General José Cristino 77, 20921-400, Rio de Janeiro, Brazil \\ email: drake@on.br \\ ${ }^{2}$ Sobolev Astronomical Institute, St. Petersburg State University, Universitetsky pr. 28, \\ Petrodvorets, 198504, St. Petersburg, Russia \\ ${ }^{3}$ Crimean Astrophysical Observatory, Nauchny, Crimea, 334413, Ukraine \\ email: polo@crao.crimea.ua \\ ${ }^{4}$ Department of Astronomy, Trieste University, Via Tiepolo 11, 34131 Trieste, Italy \\ email: hack@ts.astro.it
}

\begin{abstract}
We report the results of the spectral monitoring of the chemically peculiar (CP2) star HD 3980 in the Li I $\lambda 6708$ spectral region. High spectral resolution observations $(R=88000)$ were carried out at Mount Stromlo Observatory with the 74-inch telescope and the echelle spectrograph. HD 3980 is a late type Ap $\mathrm{SrCrEu}$ star with strong double-wave photometric variations in the visible and in the near infrared. We estimated the effective temperature, surface gravity, mass, luminosity, and radius of this star. The monitoring of this star shows strong spectral variations, especially in the profile of $\operatorname{Li}$ I $\lambda 6707.8$. We studied the variations of the equivalent width and position of the Li I line as a function of the rotating phase and compared them with the magnetic field variations and light curves taken from the literature. The synchronism of the variations of the Li I line profile, light curve, and magnetic field strength can be explained in terms of the oblique magnetic rotator model. The strong variations of position and intensity of this line with the rotation phase enable us to consider this star as a new member of the intriguing group of "Li-spotted" CP stars.
\end{abstract}

Keywords. Stars: chemically peculiar, stars: Li abundance, stars: individual: (HD 3980), stars: fundamental parameters

\section{Introduction}

HD 3980 (HR 183, $\xi$ Phe) is a bright Southern cool magnetic CP2 star. Its chemical peculiarity was classified as "Sr-Cr-Eu" by Bidelman \& MacConnell (1973). Wood (1978) noted the possible presence of an unusually strong magnetic field.

We present the results of the spectral monitoring of HD 3980 in the Li I $\lambda 6708$ spectral region. Our analysis shows that this star has a very high abundances of the Rare-Earth element (REE) praseodymium and a high lithium abundance. However, the most interesting result is that the equivalent width and position of the Li I line vary with rotattional phase, suggesting that lithium is concentrated in two small areas on the stellar surface, close to the magnetic poles.

North et al. (1998) found evidence for Li-rich spots on the surface of the rapidlyoscillating Ap star HD 83368. A detailed study by Polosukhina et al. (1999, 2000) revealed a very strong $\mathrm{Li}$ I $\lambda 6708$ line with significant variations in the intensity and position with rotational phase. The behavior of this line was explained by the existence of two opposite Li-rich spots on the stellar surface using the oblique rotator model.

Application of the Doppler Imaging code INVERs12 written by N. Piskunov to HD 83368 (Kochukhov et al. 2003, Kochukhov et al. 2004) permitted the reconstruction of surface 
distribution maps of several light, iron-peak and Rare-Earth elements and the correlation of these surface distributions with phase variations of the magnetic field. The abundance anomalies form spots or rings on the stellar surface, whose location is related to the magnetic field structure.

Polosukhina et al. (2003a) studied the behavior of the resonance line Li I $\lambda 6708$ in eleven CP stars. We have also observed HD 15144 whose physical atmospheric parameters $\left(T_{\text {eff }}\right.$ and $\left.\log g\right)$ are similar to those of HD 3980. But, only one star from the sample, HD 3980, has a strong and variable Li I line (Polosukhina et al. 2003b). Faraggiana et al. (1996) found the first indication of the variability of Li I $\lambda 6708$ in this star. Two sets of spectra, taken on even and on odd Julian days, showed a shift in wavelength for this line.. But the spectra were obtained at two phases only, which prevented these authors from studying any correlation with the light curve.

\section{Observations}

High spectral resolution observations $(R=88000)$ were carried out at Mount Stromlo Observatory (Australia) during an observing run in September-October 2001 with the 74inch telescope and the coudé echelle spectrograph with the 32 -inch camera. An echelle grating with $31.6 \mathrm{l} \mathrm{mm}^{-1}$ and a $2 \mathrm{~K} \times 4 \mathrm{~K}$ CCD detector were used. The spectra were reduced with standard IRAF procedures consisting of bias substraction, flat-field normalization, and extraction of individual orders. Th-Ar comparison spectra were obtained in the beginning and in the end of each night.

\section{Stellar parameters}

The Hipparcos distance to HD 3980 is $65.4 \pm 2.5$ pc. Assuming no interstellar reddening due to its proximity, and using a Bolometric Correction of $B C=-0.02$ calculated using the relation of Stȩpień (1994) for Ap stars, we found $M_{\mathrm{V}}=1.63 \pm 0.08$ and $\log L / L_{\odot}=1.249 \pm 0.032$.

To estimate the effective temperature of HD 3980, we used photometry in the Strömgren and Geneva systems and an analysis of the $\mathrm{H} \alpha$ profile. Strömgren photometry (Vogt \& Faúndez 1979) with 12 absolute measurements showed that the star is variable. However, the observations are insufficient for a reliable period determination. A detailed analysis of the photometric and the magnetic variability of HD 3980 (Maitzen et al. 1980) found a period of $3.9516 \pm 0.0003$ days. The visible light curves show a double wave with different amplitudes, the maximum amplitude occurs in $v$ where the primary minimum depth is $0.13 \mathrm{mag}$ and the secondary minimum is half of this value. Both maxima are nearly equal and all extrema are separated by a quarter of the rotational period.

Catalano et al. (1998) studied HD 3980 in the near infrared at 1.25, 1.6, and $2.2 \mu \mathrm{m}$ This star is variable in the infrared with the same period as in the visible, although with smaller amplitudes $(\sim 0.03 \mathrm{mag}$ in all three filters $)$.

Calibrations of effective temperature using Strömgren uvby $\beta$ photometry were obtained by Napiwotzki et al. (1993) for normal and chemically peculiar A stars. However, the temperature calibrations for Ap stars based on the photometric indices $[u-b]$ and $\left[c_{1}\right]$, were derived only for stars hotter than HD 3980 ( $\left.T_{\text {eff }} \geqslant 8400 \mathrm{~K}\right)$. Thus, temperature calibration for normal A stars with the $T_{\text {eff }} \leqslant 8400 \mathrm{~K}$ was used for the temperature determination. Using the value $(b-y)_{0}=0.070$ (Hauck \& Mermilliod 1998) we derived for HD $3980 T_{\text {eff }}=8180 \mathrm{~K}$, whereas the value of $(b-y)_{0}=0.089$ taken from Vogt et al. (1998) results in $T_{\text {eff }}=8000 \mathrm{~K}$.

Hauck \& North (1993) discussed the determination of the effective temperature of 


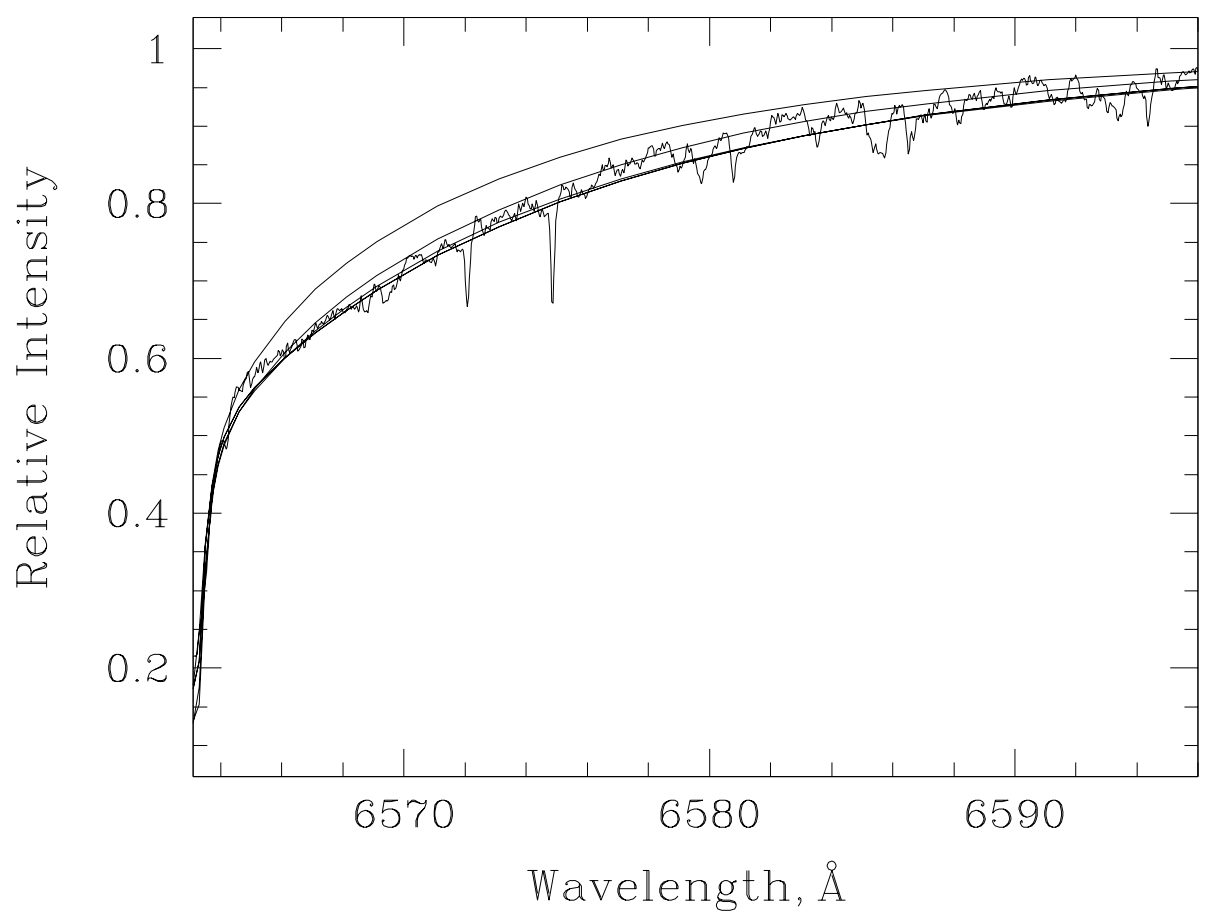

Figure 1. The H $\alpha$ profile compared with theoretical profiles (Kurucz 1979) for effective temperatures (from top to bottom) $T_{\text {eff }}=7500,8000$, and $8500 \mathrm{~K}$ and $\log g=4.0$.

different kinds of chemically peculiar stars from Geneva photometry. Using their relation between the effective temperature and the $(B 2-G)$ index, we derived $T_{\text {eff }}=8236 \mathrm{~K}$, where $(B 2-G)=-0.487$ was from Rufener (1988).

The slope of the wings of $\mathrm{H} \alpha$ is a good indicator of the effective temperature for Atype stars. We compared the observed $\mathrm{H} \alpha$ line profile of HD 3980 with the theoretical profiles calculated by Kurucz (1979). As can be seen in Fig. 1, the $\mathrm{H} \alpha$ line wings match temperatures between $T_{\text {eff }}=7500$ and $8000 \mathrm{~K}$. Nevertheless, the curvature of the echelle spectra does not permit one to compare the profile of the broad $\mathrm{H} \alpha$ line with high accuracy.

Taking into account that magnetic Ap stars are generally bluer than normal stars with the same effective temperature (North et al. 1997). This results in an enhancement of the photometrically estimated effective temperature. We adopted $T_{\text {eff }}=8000 \pm 250 \mathrm{~K}$.

Using the HR diagram (Fig. 2) we derived the following values of the stellar mass, surface gravity, and radius: $M=1.93 \pm 0.05 M_{\odot}, \log g=4.04 \pm 0.05$, and $R / R_{\odot}=$ $2.11 \pm 0.13$.

\section{Li spots}

Figure 3 shows spectra of HD 3980 in the Li I $\lambda 6708$ spectral region. The rotational phases of HD 3980 were calculated with the ephemeris from Maitzen et al. (1980). The position and intensity of the Li I line vary over the rotating phase. The strong displacement of the Li I line, about $\pm 0.3 \AA$, as well as its intensity variations, suggest that HD 3980 has an inhomogeneous Li abundance distribution. Two "Li spots" are observed on its surface. In our interpretation, the Li I line originating in spot 1 appears at phase $\varphi=-0.168 \equiv 0.832$ and moves to the red until phase $\varphi=0.146$. The second Li-rich spot 


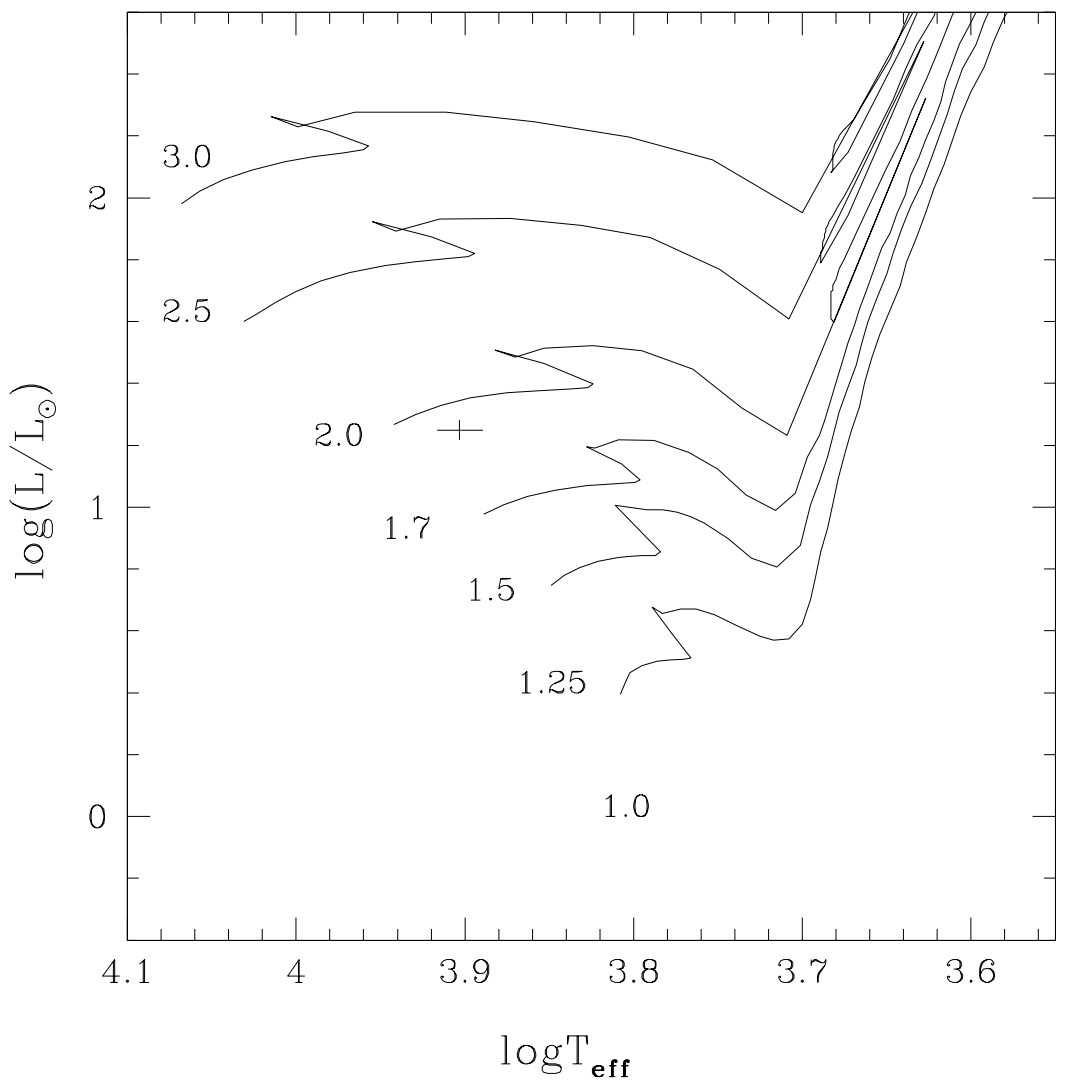

Figure 2. The Hertzsprung-Russell diagram with the position of HD 3980 (cross). The size of the symbol shows the error bars on estimated luminosity and temperature. The evolutionary tracks were taken from Schaller et al. (1992). Numbers near the tracks indicate the corresponding Main-Sequence masses in solar units.

(spot 2) appears by phase 0.319 and is observed until phase $\varphi=0.625$. The line of Pr III $\lambda 6706.705$ near the Li feature, which is a strong indicator of roAp stars, is clearly visible in the spectra.

In Fig. 4 we show the variations with rotating phase of the equivalent width and position of Li I $\lambda 6708$, as well as the magnetic field variations and the light curve from Maitzen et al. (1980). The variations of the equivalent width and position of the Li I line were approximated by a sinusoidal law. The mean position of the Li I line is $\lambda 6708.00$ and the corresponding radial velocity variations are $\pm 17.9 \mathrm{~km} \mathrm{~s}^{-1}$ for spot 1 and $\pm 15.6 \mathrm{~km} \mathrm{~s}^{-1}$ for spot 2. The mean value of the equivalent width is $73 \mathrm{~m} \AA$, and the amplitude of its variation is $\pm 27 \mathrm{~m} \AA$. The correlation $\dagger$ between the variations of the Li I line profile and

$\dagger$ Comparison of the $\mathrm{Li}$ line position and the intensity variations with a light curve shows that maximum equivalent width of $\mathrm{Li}$ I $\lambda 6708$ occurs slightly earlier $(\sim 0.13$ of rotational phase $)$ than the minima of the light curve. It may be caused by the long epoch difference (about 27 years) between photometric and spectral observations. A small uncertainty in the rotational ephemeris could result in such discrepancy. The best coincidence between the minima of the light curve and the maxima of the Li line intensity can be achieved with $P=3.9514$ days. Nevertheless, since lithium is concentrated in small areas close to the magnetic poles, it is worth noting that Kurtz et al. (1992) found that mean light variations of HD 83368 lag behind the magnetic field variations by $\sim 0.06$ of the rotational phase. However, new less time-separated observations are needed for HD 3980 to clarify this question. 


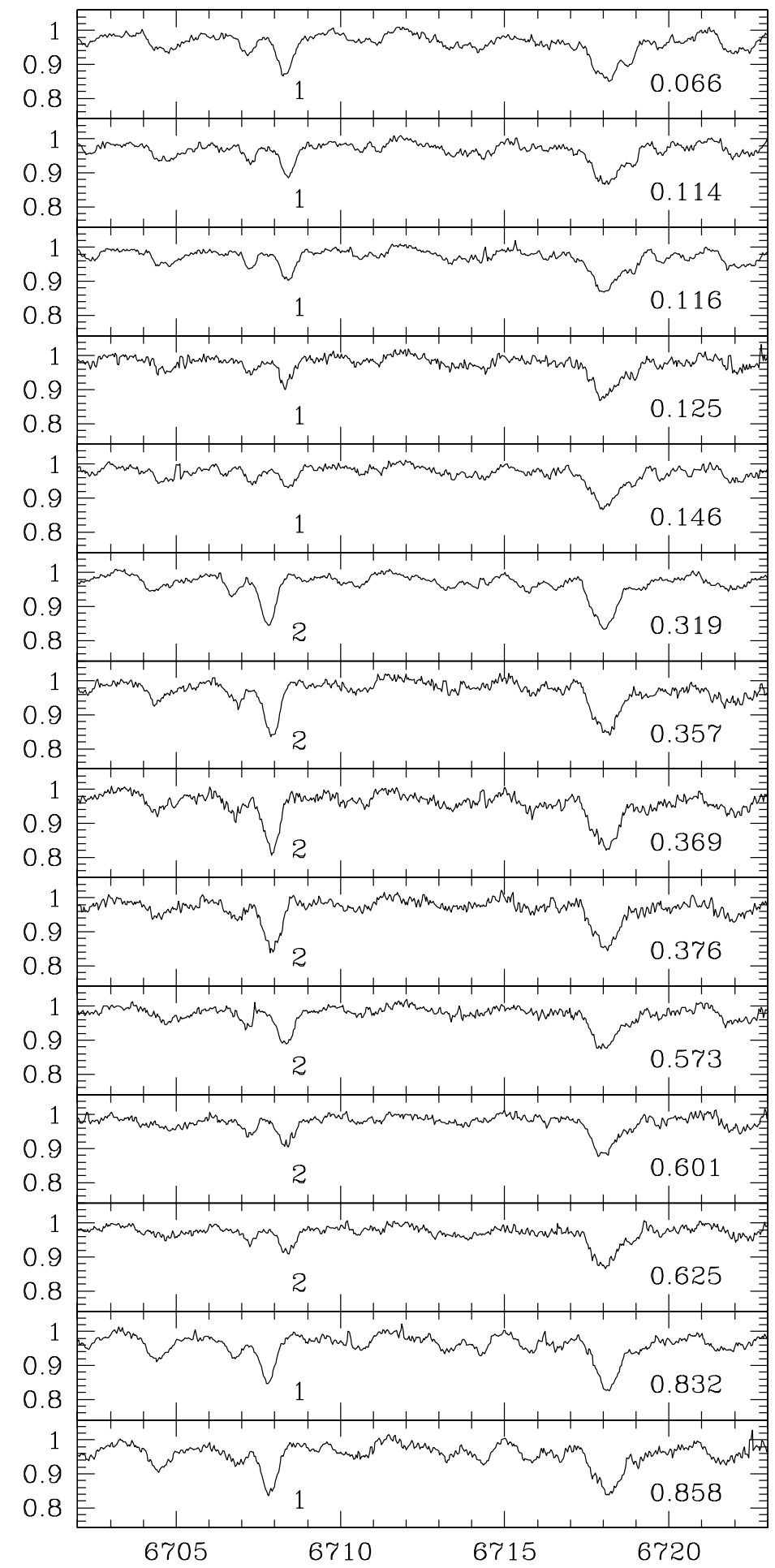

Wavelength, $\AA$

Figure 3. Spectra of HD 3980 near Li I $\lambda 6708$. The rotational phases are given on the right. The Li I line shows strong variability, both in position and in intensity. Numbers near Li I $\lambda 6708$ show the origin of this line from spot 1 or 2 . 


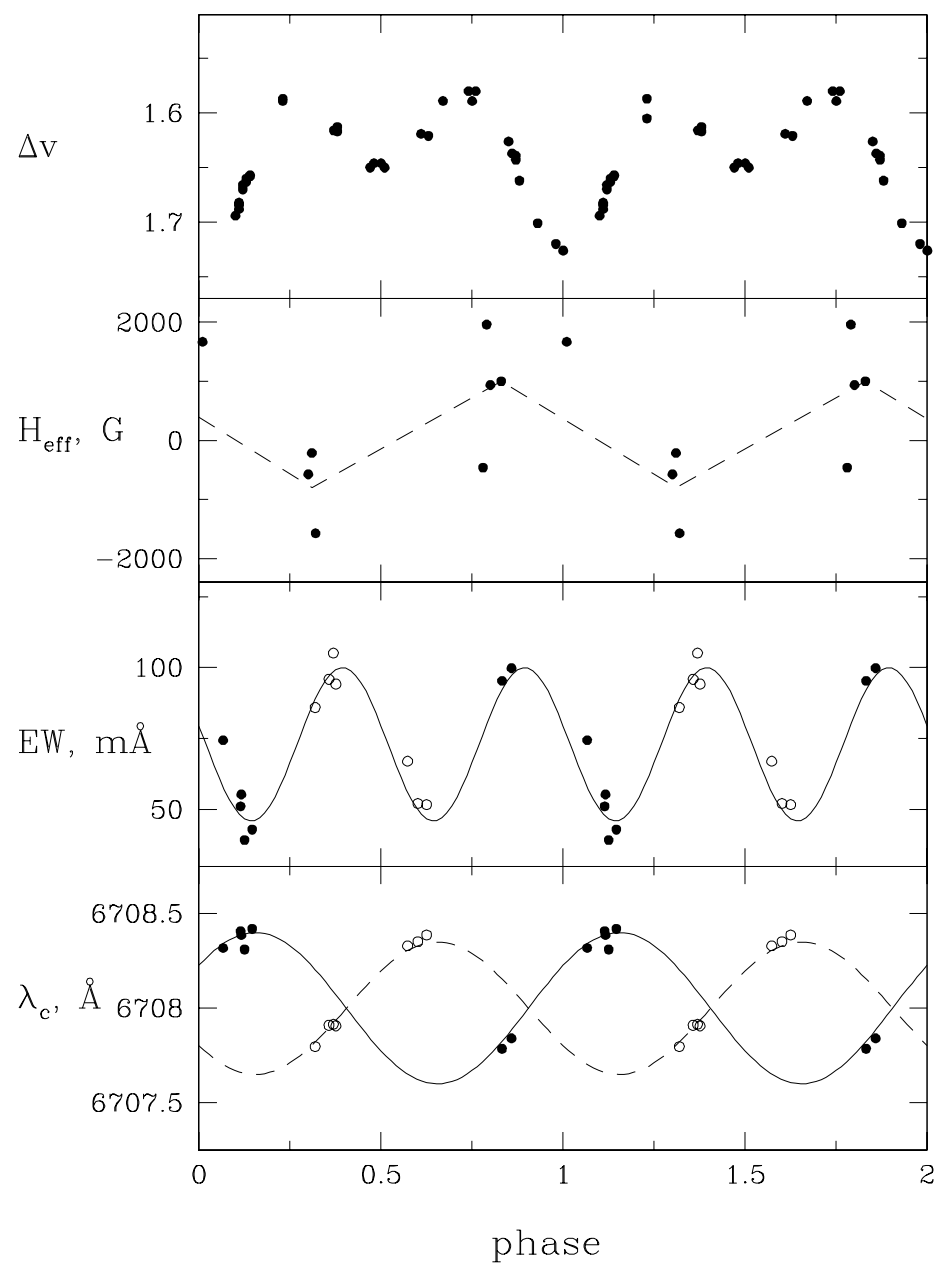

Figure 4. HD 3980. From top to bottom: photometric and magnetic field variations (from Maitzen et al. 1980), equivalent width and position variations of Li I 6708 with phase. Filled and open circles on both bottom figures correspond, respectively, to spot 1 and spot 2 .

the magnetic field strength can be explained in terms of a spotted oblique rotator model (Stibbs 1950). Unfortunately, insufficient data of the magnetic field strength variations do not allow us to determine the obliquity of the magnetic axis.

The analysis of the Li I $\lambda 6708$ line profiles requires allowance for blending with lines of REE. The lines of Pr III and Ce II are especially important. The identification of two features, at $\lambda 6706.70$ and $\lambda 6727.67$ with PrIII lines was made by Polosukhina et al. (2000). We use the positions, oscillation strengths, and lower excitation potentials of the REE lines from the D.R.E.A.M. database (Palmeri et al. 2000). The atmosphere model was taken from Kurucz (1993). As an example, in Fig. 5 we present the results of spectral synthesis in the region of the Pr III and Li I lines done with the current version (April 2002) of the MOOG code (Sneden 1973) for two phases. The first phase, $\varphi=0.146$, corresponds to the disappearance of spot 1 , and the second one, $\varphi=0.319$, corresponds to spot 2 approaching the stellar central meridian. Synthetic spectra were calculated with the projected rotational velocity of $v \sin i=15 \mathrm{~km} \mathrm{~s}^{-1}$ which fits better the observed Li I line profiles. As we have already mentioned the Li I line originates from small spots 

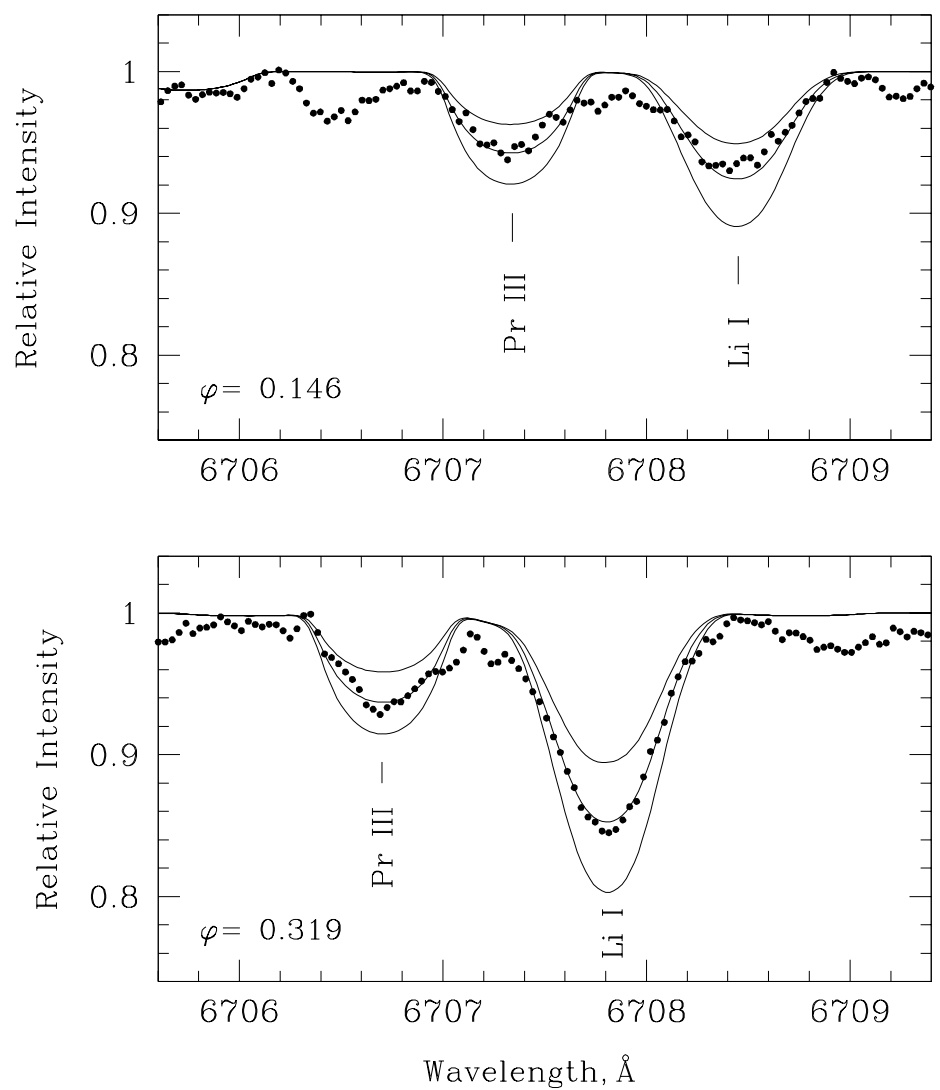

Figure 5. Observed (points) and calculated (lines) profiles of the Li I and Pr III lines at the phases $\varphi=0.146$ and $\varphi=0.319$. The synthetic spectra were calculated for lithium abundance of $\log \varepsilon(\mathrm{Li})=3.6,3.8$ and 4.0 and praseodymium abundance of $\log \varepsilon(\operatorname{Pr})=3.8,4.1$ and 4.4 for the phase $\varphi=0.146$, and $\log \varepsilon(\mathrm{Li})=4.0,4.2$ and 4.4 and $\log \varepsilon(\operatorname{Pr})=3.9,4.2$ and 4.5 for the phase $\varphi=0.319$. The best-fitting spectrum corresponds to $\log \varepsilon(\mathrm{Li})=3.8$ and $\log \varepsilon(\operatorname{Pr})=4.1$ for the phase $\varphi=0.146$ and $\log \varepsilon(\operatorname{Li})=4.2$ and $\log \varepsilon(\operatorname{Pr})=4.2$ for the phase $\varphi=0.319$.

on the stellar surface, and, in this manner, it is less affected by stellar rotation. We found that the praseodymium abundance is strongly enhanced relative to the solar value: $[\mathrm{Pr} / \mathrm{H}]=3.5$ where $[\mathrm{X} / \mathrm{H}]=\log \varepsilon(\mathrm{X})_{\text {star }}-\log \varepsilon(\mathrm{X})_{\odot}$ and $\left.\log \varepsilon(\mathrm{X})=\log \varepsilon\left(N_{\mathrm{X}} / N_{\mathrm{H}}\right)+12.0\right)$. Lithium shows a strong overabundance relative to the interstellar medium. The value $\log \varepsilon(\mathrm{Li})=3.8$ was found at phase $\varphi=0.146$, and $\log \varepsilon(\mathrm{Li})=4.2$ for phase $\varphi=0.319$, the Li I line being observed near its maximum intensity. These values of Li abundance were obtained using the assumption that the $\mathrm{Li}$ I line originates from the whole stellar disc. However, the variations in the position and the intensity of the Li I line indicates that it is connected with two relatively small regions on the stellar surface. Thus, the derived value of $\log \varepsilon(\mathrm{Li})=4.2$ is a lower limit of the $\mathrm{Li}$ abundance in the Li-rich region of the stellar surface. Doppler Imaging technique might be applied for mapping the Li distribution on the stellar surface and determination of the local abundances of this element. 


\section{Conclusions}

A good correlations between the positions of the spots, the magnetic field and the brightness indicates a possible connection between the magnetic field configuration, the local structure of the atmosphere and the distribution of the chemical anomalies.

A large overabundance of praseodymium can be explained by the process of chemical diffusion (Michaud 1970). Strong magnetic fields affect the diffusing ions, causing significant anisotropy in the processes of chemical transport. In this way, the diffusion hypothesis accounts qualitatively for overabundances of the Fe-peak, and Rare-Earth elements, since these elements have many absorption lines near the flux maximum at the temperature of the radiative zone, and, hence, they are driven upwards to the surface (Kurtz \& Martinez 2000). The ambipolar diffusion of hydrogen which affects much more strongly light ionized particles may enhance significantly the Li abundance and might also enhance the ${ }^{6} \mathrm{Li} /{ }^{7} \mathrm{Li}$ isotopic ratio (Babel \& Michaud 1991, Babel 1993). Another probable mechanism could be Li production by spallation reactions on the stellar surface in the presence of strong magnetic fields. In this case, the ${ }^{6} \mathrm{Li} /{ }^{7} \mathrm{Li}$ ratio would be enhanced. Nevertheless, a reliable determination of the Li isotopic ratio in magnetic CP stars is a very difficult task due to a blending of the red wing of the Li I line with two faint REE lines (Nd II $\lambda 6708.03$ and Ce II $\lambda 6708.10)$ whose $\log g f$ values, as well as the abundances of $\mathrm{Nd}$ and $\mathrm{Ce}$, cannot be derived with a sufficiently high precision.

The investigation of the physical conditions in Li spots should be the next step in the study of the cool magnetic CP stars. Lithium might be the key element to improve our understanding of the atmospheric structure of these enigmatic stars.

\section{Acknowledgements}

N.A.D. and N.S.P. are grateful for the warm hospitality received during the observing run at the Mount Stromlo Observatory. Special thanks to Drs. Peter Wood, Simon O'Toole and Ivan Baldry. N.A.D. thanks financial support of MCT (381.744/00-0) and computing support of FAPERJ (E-26/171.647/99). SIMBAD and VALD services have been used in this work.

\section{References}

Babel, J., Michaud, G. 1991, A\&\&A, 248, 155

Babel, J. 1993, in ASP Conf. Ser. 44, Peculiar versus Normal Phenomena in A-type and Related Stars, eds. M.M. Dworetsky, F. Castelli, \& R. Faraggiana, 458

Bidelman, W.P., MacConnell, D.J. 1973, AJ, 78, 687

Catalano, F.A., Leone, F., Kroll, R. 1998, A\&AS, 129, 463

Faraggiana, R., Gerbaldi, M., Delmas, F. 1996, Ap\&SS, 238, 169

Hauck, B., North, P. 1993, A\&A, 269, 403

Hauck, B., Mermilliod, M. 1998, A\&SAS, 129, 431

Kochukhov, O., Drake, N.A., de la Reza, R. 2003, CD-ROM, in ASP Conf. Ser., Modeling of Stellar Atmospheres, eds. N.E. Piskunov, W.W. Weiss, \& D.F. Gray

Kochukhov, O., Drake, N.A., Piskunov, N., de la Reza, R. 2004, A\&\&A (in press)

Kurtz, D.W., Kanaan, A., Martinez, P., Triple, P. 1992, MNRAS, 255, 289

Kurtz, D.W., Martinez, P. 2000, Baltic Astronomy, 9, 253

Kurucz, R.L. 1979, ApJS, 40, 1

Kurucz, R.L. 1993, CD-ROM 13, ATLAS9 Stellar Atmosphere Programs and $2 \mathrm{~km} / \mathrm{s}$ Grid (Cambridge: $\mathrm{SAO}$ )

Maitzen, H.M., Weiss, W.W., Wood, H.J. 1980, A\&A, 81, 323

Michaud, G. 1970, ApJ, 160, 641

Napiwotzki, R., Schönberner, D., Wenske, V. 1993, A\& A, 268, 653 
North, P., Jaschek, C., Hauck, B., Figueras, F., Torra, J., Künzli, M. 1997, in Proceedings of the ESA Symposium "Hipparcos - Venice 97", ESA SP-402, 239

North, P., Polosukhina, N., Malanushenko, V., Hack, M. 1998, A\& A, 333, 644

Palmeri, P., Quinet, P., Frémat, Y., Wyart, J.F., Biémont, E. 2000, ApJS, 129, 367

Polosukhina, N., Kurtz, D., Hack, M., North, P., Ilyin, I., Zverko, J., Shakhovskoy, D. 1999, $A \mathscr{E A}, 351,283$

Polosukhina, N.S., Shavrina, A.V., Hack, M., Khalack, V., Tsymbal, V., North, P. 2000, A\&AA, 357,920

Polosukhina, N.S., Drake, N.A., Hack, M., de la Reza, R., Wood, P.R., Shavrina, A.V. 2003a, CD-ROM, in ASP Conf. Ser., Modeling of Stellar Atmospheres, eds. N.E. Piskunov, W.W. Weiss, \& D.F. Gray (astro-ph/0301150)

Polosukhina, N.S., Drake, N.A., Hack, M., de la Reza, R., Shakhovskoy, D. 2003b, in ASP Conf. Ser., Stellar Rotation, eds. A. Maeder \& P. Eenens (in press)

Rufener, F. 1988, Catalogue of stars measured in the Geneva Observatory Photometric System, 4th ed., Geneva Observatory

Schaller, G., Schaerer, D., Meynet, G., Maeder, A. 1992, A\&AS, 96, 269

Sneden, C. 1973, ApJ, 184, 839

Stępień, K. 1994, in Chemically Peculiar and Magnetic Stars, ed. J. Zverko \& J. Ziznovsky, 8

Stibbs, D.W.N. 1950, MNRAS, 110, 395

Vogt, N., Faúndez A., M. 1979, A\&AS, 36, 477

Vogt, N., Kerschbaum, F., Maitzen, H.M., Faúndez-Abans, M. 1998, A\&AS, 130, 455

Wood, H.J. 1978, BAAS, 10, 635 